antibiotic therapy in $\mathrm{H}$. pylori-negative patients has yet to be clarified.

Original article Raderer M et al. (2006) Successful antibiotic treatment of Helicobacter pylori negative gastric mucosa associated lymphoid tissue lymphomas. Gut 55: 616-618

\section{Hypnosis shows efficacy in treating functional gastrointestinal disorders}

Disorders such as noncardiac chest pain and functional dyspepsia are known to be associated with psychological factors that aggravate the patient's distressing physical symptoms. Medical treatments are ineffective for many patients, especially those suffering from noncardiac chest pain. Hypnotherapy has been suggested as an alternative treatment that could provide lasting improvements in symptoms, and might also reduce medication use. The role of hypnotherapy in treating functional gastrointestinal disorders has now been strengthened by two studies that demonstrate its short-term efficacy in treating functional dyspepsia and noncardiac chest pain, respectively.

Jones et al. enrolled patients with anginalike, noncardiac chest pain whose coronary angiography results were normal, and in whom esophageal reflux had been ruled out as a cause of their symptoms. After a 4-week baseline period, patients were randomly allocated to receive hypnotherapy $(n=12)$ or supportive listening $(n=13)$. Both groups received 1430 min treatment sessions during 17 weeks (the frequency of treatment declined from weekly to monthly over this period). Patients who received supportive listening (controls) also received placebo medication, to assist with compliance and maximize the patients' expectations of benefit. Hypnotherapy offered clear benefits: $80 \%$ of the hypnotherapy group showed an improvement in chest pain, compared with $23 \%$ of controls. Similarly, $73 \%$ of the hypnotherapy group reported an improvement in general well-being, compared with $23 \%$ of controls. Importantly, patients who underwent hypnotherapy reduced their medication use, whereas nonstudy medication use increased in control patients. The authors speculate that hypnosis might also be effective in patients who were not subjected to such a stringent selection process, and those with other forms of noncardiac chest pain.
Similar benefits were seen in Chiarioni et al.'s study, which compared the effect of a single hypnosis session with that of a single dose of cisapride on gastric emptying. Their study enrolled 11 healthy volunteers and 15 patients with severe, chronic dyspepsia that had not responded to prokinetic or antisecretory medications (including cisapride). Gastric emptying time after eating a standard meal was evaluated on three occasions (at weekly intervals) in each individual: at baseline; with $10 \mathrm{mg}$ cisapride (administered $30 \mathrm{~min}$ before the meal); and during a 90 min hypnotic trance (initiated 30 min after ingestion of the meal).

Surprisingly, cisapride reduced gastric emptying time only in dyspeptic patients (from $274 \min \pm 16.8$ at baseline to $227 \min \pm 13.2$ ) and had no effect in healthy volunteers, despite the fact that these dyspeptic patients had not previously responded to cisapride. Hypnosis shortened gastric emptying time more than cisapride in both groups (in dyspeptic patients, gastric emptying time under hypnosis was 150 min \pm 9.7 , similar to that seen in healthy volunteers). The authors suggest that the prokinetic effects of hypnosis might be caused by an overall, trance-induced decrease in the activity of the sympathetic nervous system. They speculate that improvement in the symptoms of abdominal fullness and discomfort might result from central analgesia, mediated by hypnosis-induced modulation of perception.

Both studies confirm that hypnosis can have a beneficial effect on upper gastrointestinal function, and that its use should be considered for patients who do not respond to conventional treatment.

Original articles Jones $\mathrm{H}$ et al. (2006) Treatment of noncardiac chest pain: a controlled trial of hypnotherapy. Gut [doi: 10.1136/gut.2005.086694]

Chiarioni G et al. (2006) Prokinetic effect of gut-oriented hypnosis on gastric emptying. Aliment Pharmacol Ther 23: 1241-1249

\section{Identifying patients with Crohn's disease who are at risk of osteoporosis}

Several factors associated with reductions in bone mineral density (BMD) are prevalent in patients with Crohn's disease, but opinions are divided over the extent to which osteoporosis is a clinically significant problem for 